ELECTRONIC RESEARCH ANNOUNCEMENTS OF THE AMERICAN MATHEMATICAL SOCIETY

Volume 4, Pages 35-42 (May 5, 1998)

S $1079-6762(98) 00044-4$

\title{
ORTHOGONAL HARMONIC ANALYSIS OF FRACTAL MEASURES
}

\author{
PALLE E. T. JORGENSEN AND STEEN PEDERSEN
}

(Communicated by Yitzhak Katznelson)

\begin{abstract}
We show that certain iteration systems lead to fractal measures admitting an exact orthogonal harmonic analysis.
\end{abstract}

\section{Overview}

We study properties of pairs of Borel measures on $\mathbb{R}^{d}$ simultaneously generalizing Fourier series and the Fourier transform. We show that certain fractal measures fall within the class of measures admitting generalized Fourier series.

The class of fractal measures considered in this paper are obtained from an affine iteration construction leading to self-affine measures $\mu$ with support in $\mathbb{R}^{d}$. The affine maps are determined by a given expansive $d \times d$ matrix and a finite set of translation vectors. We show that the corresponding $L^{2}$-space $L^{2}(\mu)$ has an orthonormal basis of exponentials $e^{i 2 \pi \lambda \cdot x}$, indexed by vectors $\lambda$ in $\mathbb{R}^{d}$, provided certain geometric conditions hold for the affine system.

\section{INTRODUCTION}

1.1. Segal's question. Let $\Omega$ be a Lebesgue measurable subset of $d$-dimensional Euclidean space $\mathbb{R}^{d}, d \geq 1$. Let $L^{2}\left(m_{\Omega}\right)$ be the corresponding Hilbert space of square integrable functions with the inner product

$$
\langle f, g\rangle:=\int \overline{f(x)} g(x) d m_{\Omega}(x)
$$

where $m$ denotes Lebesgue measure on $\mathbb{R}^{d}$ and $m_{\Omega}(\Delta):=m(\Omega \cap \Delta)$ is Lebesgue measure restricted to the set $\Omega$. Motivated by a question raised by I. E. Segal, and a paper [Fug74] by B. Fuglede the problem of deciding for which $\Omega$ of finite measure, the space $L^{2}\left(m_{\Omega}\right)$ admits an orthogonal basis $\left\{e_{\lambda}(x):=\exp (i 2 \pi \lambda \cdot x)\right.$ : $\lambda \in \Lambda\}$ of exponentials, has been studied; see, e.g., [Fug74], [Jor82], [Ped87], [JP92], [Ped96], [LW97]. It is known [Fug74], [Ped87] that a connected open set $\Omega$ with finite measure admits an orthogonal basis of exponentials if and only if there exists commuting (in the sense of commuting spectral projections) self-adjoint extension

Received by the editors October 13, 1997.

1991 Mathematics Subject Classification. Primary 28A75, 42B10, 42C05; Secondary 47C05, 46L55.

Key words and phrases. Spectral pair, tiling, Fourier basis, self-similar measure, fractal, affine iteration, spectral resolution, Hilbert space.

(C)1998 American Mathematical Society 
operators $H_{j}, 1 \leq j \leq d$ of the minimal partial derivative operators $-i \frac{\partial}{\partial x_{j}}$ acting on $C_{c}^{\infty}(\Omega)$, the space of all smooth functions compactly supported in $\Omega$.

When $\Omega=[0,1]^{d}$ is a cube in $\mathbb{R}^{d}$, then the class of all possible commuting extension operators was the focus of attension in [JP97a]; the results are particularly satisfying for $d \leq 3$. Domains $\Omega$ admitting extension operators satisfying a weaker form of commutativity were studied by J. Friedrich [Fri87], in the case $d=2$.

1.2. Fuglede's Conjecture. Let $\Omega$ be a Lebesgue measurable subset of $\mathbb{R}^{d}$ with finite measure. If there exists a set $\Lambda$ such that $\left\{e_{\lambda}: \lambda \in \Lambda\right\}$ is an orthogonal basis for $L^{2}\left(m_{\Omega}\right)$, then we say that $\Omega$ is a spectral set, $\Lambda$ is a spectrum, and $(\Omega, \Lambda)$ is a spectral pair. If there exists a set $T$ so that up to sets of measure zero $\{\Omega+t: t \in T\}$ is a partition of $\mathbb{R}^{d}$, then we say that $\Omega$ is a tile and $T$ is a tiling set.

Conjecture 1.1 (Spectral Set Conjecture [Fug74]). Let $\Omega$ be a set of finite nonzero measure. Then $\Omega$ is a spectral set if and only if $\Omega$ is a tile.

This conjecture is open in both directions even for $d=1$. A set $S$ which is the affine image of a set of the form $\mathbb{Z}^{d}+A$ for some finite set $A$ so that $(A-A) \cap \mathbb{Z}^{d}=\emptyset$ is called periodic or a lattice with a base.

Conjecture 1.2 (Periodic Spectral Set Conjecture [Ped97]). Let $\Omega$ be a set of finite non-zero measure. Then $\Omega$ is a spectral set admitting a periodic spectrum if and only if $\Omega$ is a tile admitting a periodic tiling set.

It is known [Fug74], [Jor82], [Ped87] that $\Omega$ is a spectral set with spectrum $\mathbb{Z}^{d}$ if and only if $\Omega$ is a tile with tiling set $\mathbb{Z}^{d}$. In the papers [Ped96] and [LW97] the Periodic Spectral Set Conjecture is reduced to certain questions about finite subsets of the integer lattice $\mathbb{Z}^{d}$. For $d=1$ some progress towards a resolution of these questions is made in [PW97]. These results support the view that certain specific classes of spectra correspond to certain corresponding classes of tiling sets. This is further confirmed by some results in [JP97a], where we show that every periodic spectrum for the cube $\Omega=[0,1]^{d}$ is also a tiling set for the cube, and conversely that any periodic tiling set for the cube is a spectrum for the cube. Very recently the periodicity hypothesis in the cube result has been removed [IP98], [LRW98].

The results mentioned in the following will be established in a series of papers by the co-authors; the first two papers in this series are [JP97a] and [JP97b].

\section{Pairs of measures}

2.1. New pairs from old pairs. While studying the problems described in Section 1 it turned out to be necessary to study spectral pairs in more general situations. For these reasons we introduced in [JP97a] the following more general formulation. Let $\mu$ and $\nu$ be Borel measures on $\mathbb{R}^{d}$. We say that $(\mu, \nu)$ is a spectral pair if the map

$$
F f(\xi):=\int f(x) \overline{e_{\xi}(x)} d \mu(x)
$$

defined for $f \in L^{1} \cap L^{2}(\mu)$, extends by continuity to an isometric isomorphism mapping $L^{2}(\mu)$ onto $L^{2}(\nu)$. It was shown in [Ped87] that if $\mu$ is the restriction of Lebesgue measure to a connected open set of infinite measure, then the connection to commuting self-adjoint extensions of the directional derivatives described in Section 1 remains valid. One of the nice features of the more general definition of a spectral pair is that if $(\mu, \nu)$ is a spectral pair, then so is $(\nu, \mu)$. 
Recall that the convolution $\mu:=\mu_{1} * \mu_{2}$ of Borel measures $\mu_{j}$ on $\mathbb{R}^{d}$ is given by

$$
\int_{\mathbb{R}^{d}} f(x) d \mu(x)=\int_{\mathbb{R}^{d}} \int_{\mathbb{R}^{d}} f\left(x_{1}+x_{2}\right) d \mu_{1}\left(x_{1}\right) d \mu_{2}\left(x_{2}\right) .
$$

We will call a convolution $\mu_{1} * \mu_{2}$ non-overlapping if the map $f\left(x_{1}, x_{2}\right):=g\left(x_{1}+x_{2}\right)$ determines an isometric isomorphism $g \rightarrow f$ mapping $L^{2}\left(\mu_{1} * \mu_{2}\right)$ onto $L^{2}\left(\mu_{1} \times \mu_{2}\right)$. The following result allows us to construct a large class of spectral pairs.

Theorem 2.1 (Convolution Theorem). Suppose $\left(\mu_{j}, \nu_{j}\right)$ are spectral pairs in $\mathbb{R}^{d}$. If $\mu_{1} * \mu_{2}, \nu_{1} * \nu_{2}$ are non-overlapping and

$$
\left(\mu_{1} \times \nu_{2}\right)\left\{\left(x_{1}, \lambda_{2}\right): x_{1} \lambda_{2} \notin \mathbb{Z}\right\}=0,
$$

then $\left(\mu_{1} * \mu_{2}, \nu_{1} * \nu_{2}\right)$ is a spectral pair.

This result generalizes results from [JP92], [JP94], and [LW97]. All known examples of spectral pairs can be generated using this result and multiplicative (see Section 4 for a definition) spectral pairs.

2.2. Which measures are possible? It turns out that the class of measures that can be part of a spectral pair is fairly limited; for example, we have

Theorem 2.2 (Uncertainty Principle). Suppose $(\mu, \nu)$ is a spectral pair. Let $f \in$ $L^{2}(\mu), f \neq 0$, and $A, B \subset \mathbb{R}^{d}$. If $\left\|f-\chi_{A} f\right\|_{\mu} \leq \varepsilon$ and $\left\|F f-\chi_{B} F f\right\|_{\nu} \leq \delta$, then $(1-\varepsilon-\delta)^{2} \leq \mu(A) \nu(B)$.

Theorem 2.3 (Local Translation Invariance). Suppose $(\mu, \nu)$ is a spectral pair and $t \in \mathbb{R}^{d}$. If $\mathcal{O}$ and $\mathcal{O}+t$ are subsets of the support of $\mu$, then $\mu(\mathcal{O})=\mu(\mathcal{O}+t)$.

M. N. Kolountzakis and J. C. Lagarias in [KL96] discuss tilings of the real line $\mathbb{R}$ by a function. Some given measurable function $f$ tiles the real line with tile set $T$ if there exists a constant $c$ such that

$$
\sum_{t \in T} f(x+t)=c
$$

for almost every $x \in \mathbb{R}$. It follows from Local Translation Invariance that for such a function to come from a spectral pair it must be a multiple of the characteristic function of some set. In particular, a natural but naive generalization of the Spectral Set Conjecture to the setting of [KL96] is false.

The following result establishes a direct connection to the spectral pairs discussed in Section 1.

Theorem 2.4. Suppose $(\mu, \nu)$ is a spectral pair. If $\mu\left(\mathbb{R}^{d}\right)<\infty$, then $\nu$ is a counting measure with uniformly discrete support.

\section{Fractal measures}

3.1. Dual iteration systems. Consider a triplet $(R, B, L)$ such that $R$ is an expansive $d \times d$ matrix with real entries, and $B$ and $L$ are subsets of $\mathbb{R}^{d}$ so that

$$
\begin{aligned}
N & :=\# B=\# L \\
R^{n} b \cdot l & \in \mathbb{Z}, \text { for any } n \in \mathbb{N}, b \in B, l \in L \\
H_{B, L} & :=N^{-1 / 2}\left(e^{i 2 \pi b \cdot l}\right)_{b \in B, l \in L} \text { is a unitary } N \times N \text { matrix. }
\end{aligned}
$$


We introduce two dynamical systems,

$$
\begin{aligned}
\sigma_{b}(x) & :=R^{-1} x+b, \\
\tau_{l}(x) & :=R^{*} x+l,
\end{aligned}
$$

and the corresponding "attractors",

$$
X_{\sigma}:=\left\{\sum_{k=0}^{\infty} R^{-k} b_{k}: b_{k} \in B\right\}
$$

and

$$
\mathcal{L}=X_{\tau}:=\left\{\sum_{k=0}^{n} R^{* k} l_{k}: n \in \mathbb{N}, l_{k} \in L\right\} .
$$

The set $X_{\sigma}$ is the support of the unique probability measure solving the equation

$$
\mu=N^{-1} \sum_{b \in B} \mu \circ \sigma_{b}^{-1}
$$

Our goal is to show that under appropriate assumptions the exponentials $\left\{e_{\lambda}\right.$ : $\lambda \in \mathcal{L}\}$ form an orthogonal basis for $L^{2}(\mu)$. It follows from the assumptions (3.1)(3.3) on $(R, B, L)$ that the exponentials $\left(e_{\lambda}\right)_{\lambda \in \mathcal{L}}$ are orthogonal, so the question is whether or not they span all of $L^{2}(\mu)$. If we set

$$
\chi_{B}(t):=N^{-1} \sum_{b \in B} e_{b}(t),
$$

then expansiveness of $R$ implies that we have an explicit formula for the Fourier transform of $\mu$,

$$
\widehat{\mu}(t):=\int \overline{e_{t}(x)} d \mu(x)=\prod_{k=0}^{\infty} \chi_{B}\left(R^{*-k} t\right)
$$

the convergence being uniform on bounded subsets of $\mathbb{R}^{d}$. To facilitate the discussion we introduce the function

$$
Q(t):=\sum_{\lambda \in \mathcal{L}}|\widehat{\mu}(t-\lambda)|^{2}, \quad t \in \mathbb{R}^{d}
$$

and the operator $C$ given by

$$
(C q)(t):=\sum_{l \in L}\left|\chi_{B}(t-l)\right|^{2} q\left(\rho_{l}(t)\right)
$$

where $\rho_{l}(x):=R^{*-1}(x-l)$. The attractor

$$
X_{\rho}:=\left\{\sum_{k=1}^{\infty}-R^{*-k} l_{k}: l_{k} \in L\right\}
$$

corresponding to the system of $\rho_{l}$ 's will be used below. 
3.2. Orthogonal bases. Let $H_{2}(\mathcal{L})$ denote the subspace of $L^{2}(\mu)$ spanned by the orthonormal set $\left\{e_{\lambda}: \lambda \in \mathcal{L}\right\}$. Any $e_{t}, t \in \mathbb{C}^{d}$, is in $L^{2}(\mu)$, so $H_{2}(\mathcal{L})$ is a subspace of $L^{2}(\mu)$. We will show that $H_{2}(\mathcal{L})=L^{2}(\mu)$ for certain systems $(R, B, L)$ satisfying (3.1)-(3.3).

Let $Y$ denote the convex hull of the attractor $X_{\rho}$ given by (3.9), let $\|q\|_{\infty}:=$ $\sup _{y \in Y}|q(y)|$ and

$$
\|q\|_{Y, \infty}:=\left\||\nabla q|_{2}\right\|_{\infty}
$$

where $|z|_{2}:=\left(\sum_{j=1}^{d}\left|z_{j}\right|^{2}\right)^{1 / 2}$ is the usual Hilbert norm on $\mathbb{C}^{d}$. We begin by showing that, if the operator norm of $C$ acting on a suitable set of smooth functions is less than one, then $\mu$ has the basis property.

Theorem 3.1. Let $(R, B, L)$ be a system in $\mathbb{R}^{d}$ satisfying (3.1)-(3.3), $0 \in L$. Let $C$ be the operator given by (3.8), let $Y$ denote the convex hull of the attractor $X_{\rho}$ given by (3.9), and let $\|q\|_{Y, \infty}$ be given by (3.10). Suppose $L$ spans $\mathbb{R}^{d}$; if there exists $\gamma<1$ so that $\|C q\|_{Y, \infty} \leq \gamma\|q\|_{Y, \infty}$ for all $q$ in a set of $C^{1}$-functions containing $1-Q$, then $H_{2}(\mathcal{L})=L^{2}(\mu)$.

The following result allows us to compute an operator norm bound for $C$ in terms of the data $(R, B, L)$ in a fairly straightforward manner.

Theorem 3.2. Let $(R, B, L)$ be a system in $\mathbb{R}^{d}$ satisfying (3.1)-(3.3), $0 \in$ L. Let $C$ be the operator given by (3.8), and let $Y$ denote the convex hull of the attractor $X_{\rho}$ given by (3.9). If $\|q\|_{Y, \infty}$ is given by (3.10) and

$$
\beta:=2 \pi \operatorname{diam}(B) \max _{\substack{b, b^{\prime} \in B \\ l \in L}}\left\|\sin \left(2 \pi\left(b-b^{\prime}\right)(\cdot-l)\right)\right\|_{\infty},
$$

then we have

$$
\|C q\|_{Y, \infty} \leq\left[(N-1)^{2} N^{-1} \beta\left\|R^{-1}\right\|_{o p} \max _{l \in L}|l|_{2}+\left\|R^{-1}\right\|_{h s}\right]\|q\|_{Y, \infty}
$$

for any $C^{1}$-function $q$ such that $q(0)=0$. Here $\|T\|_{o p}$ is the operator norm and $\|T\|_{h s}:=\left(\sum_{j, k=1}^{d}\left|t_{j, k}\right|^{2}\right)^{1 / 2}$ is the Hilbert-Schmidt norm of a $d \times d$ matrix $T$.

As a consequence of Theorem 3.1 and Theorem 3.2 we have

Corollary 3.3. Let $(R, B, L)$ satisfy (3.1)-(3.3) and for $r \in \mathbb{N}$ let

$$
\mathcal{L}_{r}:=\left\{\sum_{k=0}^{n}\left(r R^{*}\right)^{k} l_{k}: n \in \mathbb{N}, l_{k} \in L\right\},
$$

and let $\mu_{r}$ be the probability measure solving

$$
\mu_{r}=N^{-1} \sum_{b \in B} \mu_{r} \circ \sigma_{r, b}^{-1}
$$

where $\sigma_{r, b}(x):=(r R)^{-1} x+b$. If $L$ spans $\mathbb{R}^{d}$ and $0 \in L$, then $\left\{e_{\lambda}: \lambda \in \mathcal{L}_{r}\right\}$ is an orthonormal basis for $L^{2}\left(\mu_{r}\right)$ provided $r$ is sufficiently large.

R. S. Strichartz obtained an asymptotic harmonic analysis for the class of measures considered in this paper; see [Str94] for a survey of some of Strichartz's work on self-similarity in harmonic analysis. 


\section{Applications}

4.1. The Hardy space connection. One way to construct systems $(R, B, L)$ satisfying (3.1)-(3.3) is to pick $R, B$ and $L$ so that

$$
R \in M_{d}(\mathbb{Z}), \quad R B \subset \mathbb{Z}^{d}, \quad L \subset \mathbb{Z}^{d} .
$$

In fact (4.1) implies (3.2) since $R^{n} b \cdot l=R b \cdot R^{*(n-1)} l$ for $n=1,2,3, \ldots$ The only condition that is hard to satisfy is (3.3). This condition is notoriously difficult to study; for example, it is not known which matrices with entries in the unit circle satisfy (3.3) for $N=7$; see e.g. [Haa95], [BS95] for some progress in the study of (3.3). The condition (4.1) is closely related to a condition used in the study of certain multi-dimensional wavelets. Some properties of systems $(R, B, L)$ satisfying (3.1), (4.1), and (3.3) were established in [JP96].

If $(R, B, L)$ satisfies (3.1), (4.1), and (3.3) and $z \in \mathbb{Z}^{d}$, then $\left(R, B, L_{z}\right)$ also satisfies (3.1), (4.1), and (3.3), where $L_{z}:=L+z=\{l+z: l \in L\}$. So we may often assume that $L \subset \mathbb{N}^{d}$. Therefore, if $R$ has non-negative integer entries, we will often end up with $\left\{e_{\lambda}: \lambda \in \mathcal{L}\right\}$ being an orthonormal basis for $L^{2}(\mu)$ and each element in $\mathcal{L}$ only having non-negative coordinates. This is an interesting situation because the basis property leads to

$$
f=\sum_{\lambda \in \mathcal{L}}\left\langle e_{\lambda} \mid f\right\rangle_{\mu} e_{\lambda}
$$

for $f \in L^{2}(\mu)$, so setting $z_{j}:=e^{i 2 \pi x_{j}}$ we see that

$$
f(x)=\sum_{\lambda \in \mathcal{L}}\left\langle e_{\lambda} \mid f\right\rangle_{\mu} z^{\lambda}
$$

where $z^{\lambda}:=\prod_{k=1}^{d} z_{k}^{\lambda_{k}}$; it follows that $f(x), x \in X_{\sigma}$, gives the boundary values of a function analytic in the polydisc $\left\{z \in \mathbb{C}^{d}:\left|z_{j}\right|<1\right\}$. Hence our construction shows that many fractal $L^{2}$-spaces are Hardy spaces. This is in sharp contrast to the Lebesgue spaces; for example, if $m_{[0,1]}$ is Lebesgue measure restricted to the unit interval $[0,1]$, then (essentially) the only set $\Lambda$ such that $\left\{e_{\lambda}: \lambda \in \Lambda\right\}$ is an orthonormal basis for $L^{2}\left(m_{[0,1]}\right)$ is $\Lambda=\mathbb{Z}$, and the corresponding analytic subspace spanned by $\left\{e_{\lambda}: \lambda \in \mathbb{N}_{0}\right\}$ is far from being equal to $L^{2}\left(m_{[0,1]}\right)$. Note that $m_{[0,1]}=\mu$ if $R=2, B=\{0,1 / 2\}$, and $L=\{0,1\}$.

4.2. First order operators. Let $\left(U_{t}\right)_{t \in \mathbb{R}}$ be a one-parameter group of unitary operators on some $L^{2}$-space $L^{2}(\mu)$. We say that $U_{t}$ acts multiplicatively, if

$$
U_{t}(f g)=\left(U_{t} f\right)\left(U_{t} g\right)
$$

for all $t \in \mathbb{R}$ and all $f, g \in L^{2} \cap L^{\infty}(\mu)$.

Given a probability measure admitting an orthogonal basis $\left\{e_{\lambda}: \lambda \in \Lambda\right\}$ one can define commuting extension operators $H_{j}$ by setting

$$
H_{j} e_{\lambda}:=\lambda_{j} e_{\lambda}
$$

for $\lambda \in \Lambda$. By results mentioned in Section 1 it is reasonable to think of $H_{j}$ as an "extension" of $-i \frac{\partial}{\partial x_{j}}$. Suppose further that $\mu$ is a purely singular continuous measure generated as in Section 3. One can adapt methods from [JP92] to show 
that none of the $H_{j}$ 's can satisfy Leibniz's rule, in the sense that none of the unitary groups $\left(U_{j, t}\right)_{t \in \mathbb{R}}$, given by

$$
U_{j, t}:=\exp \left(i 2 \pi H_{j} t\right)
$$

acts multiplicatively. A spectral pair is called multiplicative if the unitary groups $\left(U_{j, t}\right)_{t \in \mathbb{R}}$ act multiplicatively. We may construct a Laplace operator $\Delta$ by setting

$$
-\Delta:=H_{1}^{2}+\cdots+H_{d}^{2} \text {. }
$$

This way of constructing a Laplace operator on a fractal complements the construction considered, for example, by J. Kigami and M. Lapidus [KL93].

4.3. Examples. Using Theorem 3.2, Theorem 3.1, and equation (3.7) one can prove the following result.

Theorem 4.1. Suppose $d=1, N=2, B=\{0, a\}$, with $a \in \mathbb{R} \backslash\{0\}, R$ is an integer with $|R| \geq 2$, and $\mu$ is given by (3.5). If $R$ is odd, then $L^{2}(\mu)$ does not have a basis of exponentials for any $a \in \mathbb{R} \backslash\{0\}$. If $R$ is even and $|R| \geq 4$, then $L^{2}(\mu)$ has a basis of exponentials for any $a \in \mathbb{R} \backslash\{0\}$.

Using the Convolution Theorem (Theorem 2.1) and Theorem 4.1 one can verify the following example.

Example 4.2. Let $\mu_{0}$ be the probability measure solving (3.5) when $R=4$ and $B=\{0,1 / 2\}$. Let $L=\{0,1\}$ and let $\mathcal{L}$ be given by (3.4). Set $\Omega:=[0,1]+\mathcal{L}$. If

$$
\mu(\Delta):=m(\Delta \cap \Omega)
$$

and

$$
\nu(\Delta):=\sum_{k=-\infty}^{\infty} \mu_{0}(\Delta+k),
$$

then $(\mu, \nu)$ is a spectral pair, and $\Omega$ is a tile with tiling set $-2 \mathcal{L}$.

This is an example of a spectral set of infinite measure whose spectrum is not periodic. This takes us full circle ending up in a situation discussed in Section 1.

\section{REFERENCES}

[BS95] G. Björck and B. Saffari, New classes of finite unimodular sequences with unimodular Fourier transforms. Circulant Hadamard matrices with complex entries, C. R. Acad. Sci. Paris, Série 1, 320 (1995), 319-324. CMP 95:09

[Fri87] J. Friedrich, On first order partial differential operators on bounded regions of the plane, Math. Nachr. 131 (1987), 33-47. MR 88j:47062

[Fug74] B. Fuglede, Commuting self-adjoint partial differential operators and a group theoretic problem, J. Funct. Anal. 16 (1974), 101-121. MR 57:10500

[Haa95] U. Haagerup, Orthogonal maximal Abelian *-subalgebras of the $n \times n$ matrices and cyclic $n$-roots, preprint, 1995, $29 \mathrm{pp}$.

[IP98] A. Iosevich and S. Pedersen, Spectral and tiling properties of the unit cube, preprint, 1998.

[Jor82] P. E. T. Jorgensen, Spectral theory of finite volume domains in $\mathbb{R}^{n}$, Adv. Math. 44 (1982), 105-120. MR 84k:47024

[JP92] P. E. T. Jorgensen and S. Pedersen, Spectral theory for Borel sets in $\mathbb{R}^{n}$ of finite measure, J. Funct. Anal. 107 (1992), 72-104. MR 93k:47005

[JP94] P. E. T. Jorgensen and S. Pedersen, Harmonic analysis and fractal limit-measures induced by representations of a certain $C^{*}$-algebra, J. Funct. Anal. 125 (1994), 90-110. MR 95i: 47067 
[JP96] P. E. T. Jorgensen and S. Pedersen, Harmonic analysis of fractal measures, Constr. Approx. 12 (1996), 1-30. MR 97c:46091

[JP97a] P. E. T. Jorgensen and S. Pedersen, Spectral pairs in Cartesian coordinates, J. Fourier Anal. Appl., to appear.

[JP97b] P. E. T. Jorgensen and S. Pedersen, Dense analytic subspaces in fractal $L^{2}$-spaces, J. Anal. Math., to appear.

[KL93] J. Kigami and M. Lapidus, Weyl's problem for the spectral distribution of Laplacians on p.c.f. self-similar sets, Commun. Math. Phys. 158 (1993), 93-125. MR 94m:58225

[KL96] M. N. Kolountzakis and J. C. Lagarias, Structure of tilings of the line by a function, Duke Math. J. 82 (1996), 653-678. MR 97d:11124

[LRW98] J. C. Lagarias, J. A. Reed, and Y. Wang, Orthonormal bases of exponentials for the $n$-cube, preprint, 1998.

[LW97] J. C. Lagarias and Y. Wang, Spectral sets and factorizations of finite Abelian groups, J. Funct. Anal. 145 (1997), 73-98. MR 98b:47011b

[Ped87] S. Pedersen, Spectral theory of commuting self-adjoint partial differential operators, J. Funct. Anal. 73 (1987), 122-134. MR 89m:35163

[Ped96] S. Pedersen, Spectral sets whose spectrum is a lattice with a base, J. Funct. Anal. 141 (1996), 496-509. MR 98b:47011a

[Ped97] S. Pedersen, Fourier series and geometry, preprint, 1997.

[PW97] S. Pedersen and Y. Wang, Spectral sets, translation tiles and characteristic polynomials, preprint, 1997.

[Str94] R. S. Strichartz, Self-similarity in harmonic analysis, J. Fourier Anal. Appl. 1 (1994), 1-37. MR 96c: 42002

Department of Mathematics, University of Iowa, Iowa City, IA 52242

E-mail address: jorgen@math.uiowa.edu

Department of Mathematics, Wright State University, Dayton, OH 45435

E-mail address: steen@math.wright.edu 